University of Texas Rio Grande Valley

ScholarWorks @ UTRGV

Mechanical Engineering Faculty Publications and Presentations

6-10-2009

\title{
Phase tunable holographic fabrication for three-dimensional photonic crystal templates by using a single optical element
}

\author{
Di Xu \\ University of Pittsburgh \\ Kevin P. Chen \\ University of Pittsburgh \\ Ahmad Harb \\ The University of Texas Rio Grande Valley \\ Daniel Rodriguez \\ The University of Texas Rio Grande Valley \\ Karen Lozano \\ The University of Texas Rio Grande Valley, karen.lozano@utrgv.edu
}

See next page for additional authors

Follow this and additional works at: https://scholarworks.utrgv.edu/me_fac

Part of the Mechanical Engineering Commons

\section{Recommended Citation}

Xu, D., Chen, K. P., Harb, A., Rodriguez, D., Lozano, K., \& Lin, Y. (2009). Phase tunable holographic fabrication for three-dimensional photonic crystal templates by using a single optical element. Applied Physics Letters, 94(23), 231116. https://doi.org/10.1063/1.3149705

This Article is brought to you for free and open access by the College of Engineering and Computer Science at ScholarWorks @ UTRGV. It has been accepted for inclusion in Mechanical Engineering Faculty Publications and Presentations by an authorized administrator of ScholarWorks @ UTRGV. For more information, please contact justin.white@utrgv.edu,william.flores01@utrgv.edu. 
Authors

Di Xu, Kevin P. Chen, Ahmad Harb, Daniel Rodriguez, Karen Lozano, and Yuankun Lin

This article is available at ScholarWorks @ UTRGV: https://scholarworks.utrgv.edu/me_fac/23 
 \\ Phase tunable holographic fabrication for three-dimensional photonic crystal templates by using a single optical element}

Cite as: Appl. Phys. Lett. 94, 231116 (2009); https://doi.org/10.1063/1.3149705

Submitted: 18 February 2009 . Accepted: 10 May 2009. Published Online: 10 June 2009

Di Xu, Kevin P. Chen, Ahmad Harb, Daniel Rodriguez, Karen Lozano, and Yuankun Lin

ARTICLES YOU MAY BE INTERESTED IN

Fabrication of large area two- and three-dimensional polymer photonic crystals using single refracting prism holographic lithography

Applied Physics Letters 86, 241102 (2005); https://doi.org/10.1063/1.1947369

Fabrication of three-dimensional polymer photonic crystal structures using single diffraction element interference lithography

Applied Physics Letters 82, 1667 (2003); https://doi.org/10.1063/1.1560860

Photonic crystals through holographic lithography: Simple cubic, diamond-like, and gyroidlike structures

Applied Physics Letters 84, 5434 (2004); https://doi.org/10.1063/1.1765734

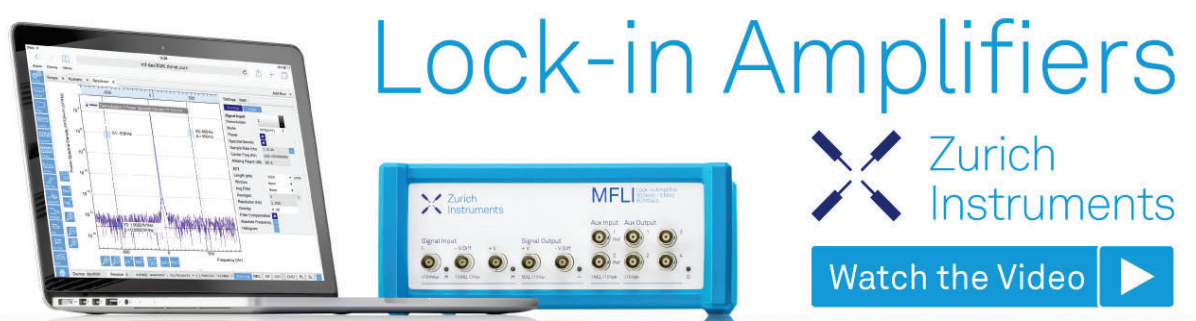




\title{
Phase tunable holographic fabrication for three-dimensional photonic crystal templates by using a single optical element
}

\author{
Di Xu, ${ }^{1}$ Kevin P. Chen, ${ }^{1, a)}$ Ahmad Harb, ${ }^{2}$ Daniel Rodriguez, ${ }^{2}$ Karen Lozano, ${ }^{2}$ and \\ Yuankun Lin ${ }^{2, b)}$ \\ ${ }^{1}$ Department of Electrical and Computer Engineering, University of Pittsburgh, Pittsburgh, \\ Pennsylvania 15261, USA \\ ${ }^{2}$ College of Science and Engineering, University of Texas-Pan American, Edinburg, Texas 78541, USA
}

(Received 18 February 2009; accepted 10 May 2009; published online 10 June 2009)

\begin{abstract}
This paper demonstrates a phase tunable holographic fabrication of three-dimensional photonic lattice structures using a single optical element. A top-cut four-side prism is employed to generate five-beam three-dimensional interference patterns. A silica glass slide is inserted into the optical path to adjust the phase of one interfering beam relative to other four beams. The phase control of the interfering laser beam renders the lattice of the interference pattern from a face-center tetragonal symmetry into a high contrast, interconnecting diamondlike symmetry. This method provides a flexible approach to fabricating three-dimensional photonic lattices with improved photonic band structures. (C) 2009 American Institute of Physics. [DOI: 10.1063/1.3149705]
\end{abstract}

Three-dimensional (3D) photonic crystal structures have been the subject of intense scientific and engineering interests since the first introduction in $1987 .^{1,2}$ The existence of a photonic bandgap in photonic crystals gives rise to a number of peculiar optical properties useful for photonic engineering. However, the fabrication of 3D photonic crystals with a complete bandgap in visible and near infrared regimes remains a great challenge. ${ }^{3}$ This is because the existing photomask lithography-based fabrication approach widely used in semiconductor industries cannot be inherited for 3D nanofabrication. Nevertheless, various approaches have been employed to fabricate photonic crystals with complex 3D periodic lattices. ${ }^{4-7}$

Among these methods, holographic laser lithography is a powerful technique to fabricate $3 \mathrm{D}$ photonic crystals. ${ }^{7,8}$ The holographic lithography constructs 3D interference patterns of multiple coherent laser beams in a photoresist to form 3D structures. The complexity and symmetry of the $3 \mathrm{D}$ photonic structures can be controlled by the number of interfering laser beams, their relative interference angles, and their respective phases.

Recently, a number of papers have demonstrated the laser holographic fabrication of 3D photonic crystal structures using a single optical element such as a refractive optical element (e.g., a top-cut prism ${ }^{9-11}$ ) or a diffractive optical element (e.g., a phase mask ${ }^{12}$ ). The optical element splits a single incoming laser beam into multiple coherent laser beams with desired interfering angles for 3D holographic pattern formation. The adaptation of single optical element in holographic lithography drastically reduces the fabrication complexity.

Technically, it is relatively easy to produce multiple coherent laser beams with precise interference angles by a single optical element. ${ }^{9-12}$ However, holographic lithography techniques using a single optical element presented so far lack precise phase control of the interfering beams. Precise phase control of interfering laser beams is critical to the fab-

\footnotetext{
${ }^{a)}$ Electronic mail: kchen@engr.pitt.edu.

${ }^{b)}$ Electronic mail: linyk@utpa.edu.
}

rication of complex, highly symmetric photonic structures such as the diamondlike structures which possess large photonic bandgaps. ${ }^{13,14}$

In this paper, we demonstrate a phase tunable holographic fabrication of 3D photonic structures using a single optical element. The technique demonstrated in this paper provides a simple approach to finely tune and optimize photonic band structures with large photonic bandgap width.

The single optical element used to construct the fivebeam interference pattern is a top-cut, four-side prism. Five beams are selected by a set of apertures from one incoming collimate beam. The use of the apertures prevents laser beams from hitting corners and edges of the prism. As shown in the inset of Fig. 1, all five laser beams are incident from the bottom side of the prism. After total internally reflected at four lateral surfaces of the prism, Beam 2-5 refract through the top surface of the prism and recombine with beam 1 to form interference patterns. To perform the phase modulation, a thin microscope glass cover slide with a uniform thickness (Corning, BK7 glass, $d=130 \mu \mathrm{m}, n=1.52$ ) is inserted into one of five beams used to form interference patterns (labeled

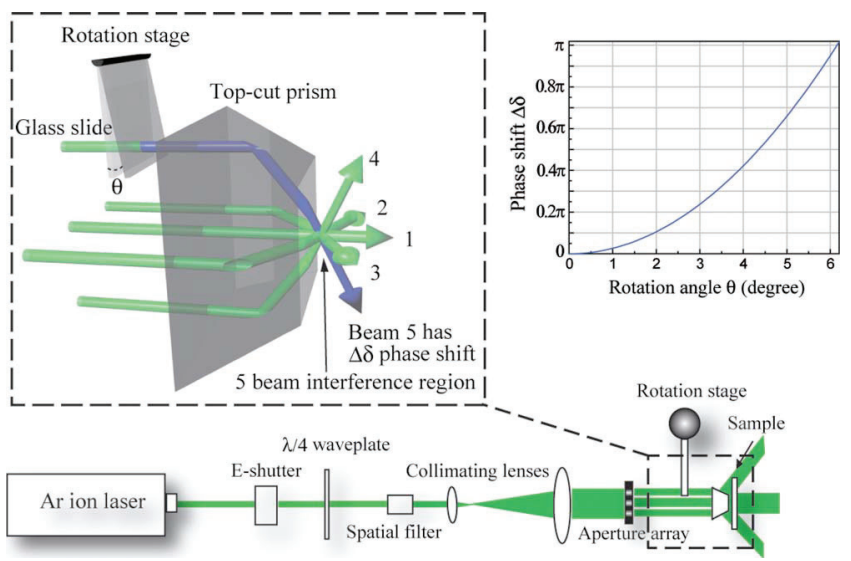

FIG. 1. (Color online) Experiment setup of the five-beam interference with one beam modulated by a glass slide. Inset: phase modulation $\Delta \delta$ as a function of the glass slide rotational angle $\theta$. 

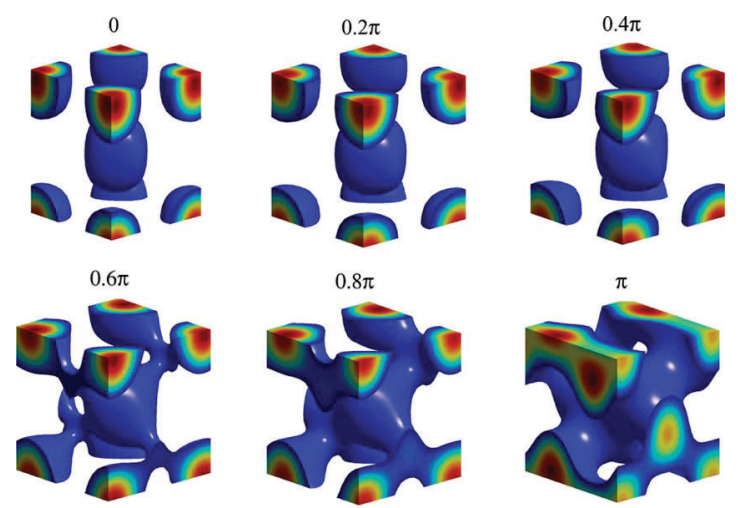

Low High

FIG. 2. (Color online) Isosurface of the unit cells of the phase modulated five-beam interference pattern, $I_{\text {mod. }}$ The phase change $\Delta \delta$ varies from 0 to $\pi$ with $0.2 \pi$ increment.

as beam 5 in Fig. 1). By rotating the glass slide, the phase of the beam 5 can be adjusted continuously.

The rationale of the phase control of one interfering beam can be briefly described as the following. The intensity profile of the five-beam interference pattern can be calculated as

$$
I=\left\langle\sum_{i=1}^{5} E_{i}^{2}\right\rangle+\sum_{i<j}^{5} E_{i} E_{j} \cos \left[\left(k_{i}-k_{j}\right) r+\Delta \delta_{i j}\right],
$$

where $E_{i}$ is electric field strength and $k_{i}$ is wave number. The insertion of the phase control glass slide in the beam 5 induces an additional phase modulation $\Delta \delta$, which only affects on the last term of Eq. (1) and we can rewrite Eq. (1) as

$$
I_{\text {mod }}=I_{\text {nonmod }}+\sum_{i=1}^{5} E_{i} E_{5} \cos \left[\left(k_{i}-k_{5}\right) r+\Delta \delta\right],
$$

where $I_{\text {mod }}$ and $I_{\text {nonmod }}$ denote the isosurface of the intensity pattern related to and not related to the phase modulation, respectively. The additional phase modulation $\Delta \delta$ as a function of the glass slide rotation angle $\theta$ can be described as

$$
\begin{aligned}
\Delta \delta(\theta)= & \frac{2 \pi}{\lambda}\left\{\frac{d}{\cos \alpha}\left[n_{\text {glass }}-n_{\text {air }} \cos (\theta-\alpha)\right]\right. \\
& \left.-\left(n_{\text {glass }}-n_{\text {air }}\right) d\right\},
\end{aligned}
$$

where $\alpha=\arcsin \left(\sin \theta / n_{\text {glass }}\right)$ and $\lambda$ is the incident wavelength. Figure 1 inset illustrates the relation between the phase change $\Delta \delta$ and the rotation angle $\theta$ from Eq. (3). From this curve, we can see that $6.1^{\circ}$ of rotation of the glass slide is enough to produce a $\pi$ phase retardation using the current setup. Figure 2 shows the variation in unit cell lattices for the five-beam interference pattern as the phase change $\Delta \delta$ evolves from 0 to $\pi$ with a $0.2 \pi$ increment. The evolution of the phase change $\Delta \delta$ renders the interference pattern (or holographic photonic crystal) from face-centered-cubic or facecentered-tetragonal (FCT) structure into interconnected structures. When the phase modulation reaching the optimal value $\Delta \delta=\pi$, a diamondlike network is formed. ${ }^{13,14}$

To experimentally validate the phase tuning and structure controlling, 3D photonic crystal templates were fabricated in a negative-toned photoresist mixture. It contains 88.93 wt $\%$ dipentaerythritol penta/hexaacrylate monomer

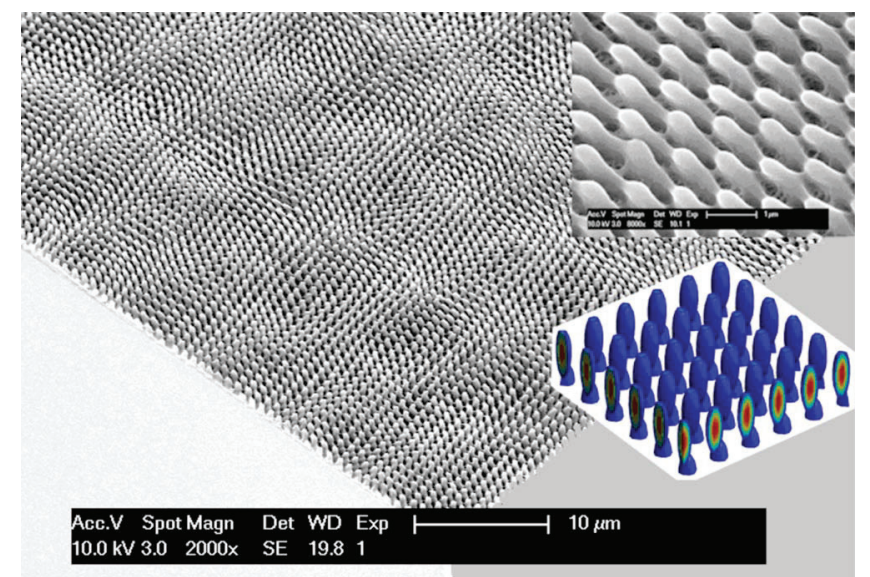

FIG. 3. (Color online) SEM pictures of fabricated structures in the photoresist through the five-beam interference without a phase modulation. Insets are the zoom in view of the bottom layer and a simulation for a comparison.

(from Aldrich), $0.22 \mathrm{wt} \%$ photoinitiator rose bengal, 0.82 wt $\%$ coinitiator $N$-phenyl glycine and 10.03 wt $\%$ chain extender $N$-vinyl pyrrolidinone. ${ }^{15}$ The mixture was spin-coated on glass slides at $2000 \mathrm{rpm}$ for $2 \mathrm{~min}$. No prebake procedure was used before receiving exposure.

A $514.5 \mathrm{~nm}$ circularly polarized laser beam from Ar ion laser (Coherent Inc.) was cleaned, expanded, and collimated by spatial filter and collimating lens. The BK7 microscope slide used for phase tuning was mounted on a rotational stage. The exposure dose in use is tested in the range of $100-1000 \mathrm{~mJ} / \mathrm{cm}^{2}$. After exposure, the photoresist samples were developed directly in propylene glycol methyl ether acetate for $20 \mathrm{~s}$, followed by rinsing in isopropanol for $10 \mathrm{~s}$ and then left to dry in air.

Figure 3 shows the scanning electric microscope (SEM) pictures for the recorded five-beam interference pattern in the photoresist. In this case, the glass slide for phase modulation was not used. Without the phase modulation, the developed photoresist did not form interconnected networks. Only one layer of developed photoresist was left on the glass slide. Two-dimensional structures shown in the SEM is in good agreement with the simulated one (see insert in Fig. 3).

In a sharp contrast, the introduction of a phase tuning mechanism in the five-beam holographic lithography using the prism yields overlapping diamondlike structures. Figure 4(a) shows the SEM image for the photonic crystal template formed in photoresist with diamondlike structures, which were achieved by the phase tuning of the beam 5. As predicted by the simulation in Fig. 4(b), the fine phase tuning transforms the photonic crystal template from the FCT symmetry to diamondlike symmetry. The interference pattern locked in photoresist clearly shows interlaced structures with diamondlike symmetries in Fig. 4. The period of diamondlike structure in the Fig. 4(a) in $x$ (or $y$ ) direction is measured to be $0.85 \mu \mathrm{m}$, in good agreement with a theoretic prediction of $0.84 \mu \mathrm{m}$ with an interference angle of $37.5^{\circ}$ and a period of $0.82 \mu \mathrm{m}$ in Fig. 3 for the fabricated one-layer structure.

A detail examination of the SEM photo shows that various interlaced structures were produced periodically on the surface of the photoresist with a period of around $8.6 \mu \mathrm{m}$, which is approximately ten times as large as the diamondlike lattice period. Zoom-in SEM pictures, as shown in Figs. 4(c)-4(f), present detailed features of various interlaced 


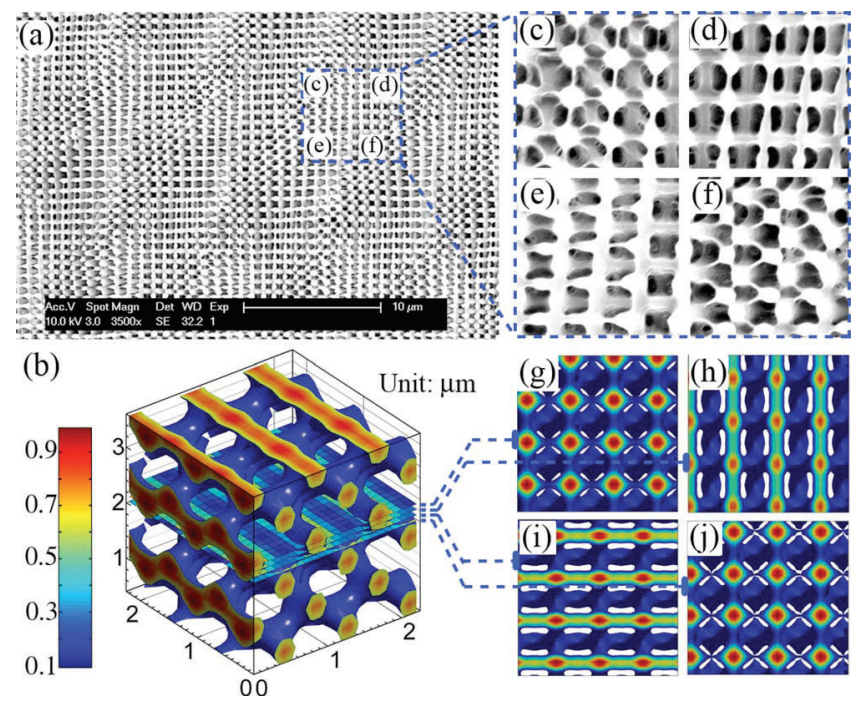

FIG. 4. (Color online) (Color Online) (a) SEM pictures for the photoresist templates of the interconnecting diamondlike structure, produced by the five-beam interference with phase retardation. (b) Computed five-beam interference pattern and its selected cross-section planes along height $(z)$ direction; [(c)-(f)] Structure variation described by the zoom in SEM views of the diamondlike template and their corresponding simulation planes from (b).

structures in the developed photoresist. These structural variations could be attributed to a slight misalignment of the sample. The surface of the photoresist film is not completely perpendicular to the normal incident laser beam, but cut through the (001) surface of the diamondlike structure at a small angle. This will result in surface topology representing diamondlike structures at different depth. The comparison between the simulation and the experiment showed in Fig. 4 confirms this speculation. Figures $4(\mathrm{~g})-4(\mathrm{j})$ shows the computed five-beam interference pattern where one beam has a phase retardation of $\pi$ relative to other four beams, and its selected cross-section planes along height $(z)$ direction. The fabricated topographic images shown in Figs. 4(c)-4(f) match well with those simulation planes.

It should be pointed out that the photonic bandgap properties of the photonic crystal can be improved by such phase modulation. Theoretical simulation predicts that the photonic structure templates duplicated from the five-beam interference patterns can have complete bandgap if the templates or their inverse replica structures are translated into highindex materials. Such inversion processes have been well-documented. ${ }^{16}$ If the template structure shown in Fig. 4 is translated into a $3 \mathrm{D}$ photonic structure consisting of air filled voids and high refractive index dielectric materials such as $\mathrm{Si}$, the phase modulation $\Delta \delta$ from 0 to $\pi$ will lead to an improvement of photonic bandgap width (bandgap width/ bandgap central frequency) from $3.8 \%$ to $25 \%$, with a change in bandgap location from band $8-9$ to band 2-3. 13,14,17 $^{\text {. }}$

In summary, we have demonstrated phase tunable holography fabrication of $3 \mathrm{D}$ diamondlike photonic crystal templates using a top-cut prism as a refractive optical element and a glass slide as a phase modulator. The phase modulation can transfer the template from FCT structure to diamondlike structure, as confirmed through SEM images. This paper provides a simple and versatile method to control holographic interference patterns to fabricate photonic crystal with optimized optical properties.

This work was supported by research grants from the U.S. National Science Foundation under Grant Nos. DMI0556086 (K.P.C.), CMMI-0609345 (Y.L. and K.L.) and DMR-0722754 (Y.L.).

${ }^{1}$ S. John, Phys. Rev. Lett. 58, 2486 (1987).

${ }^{2}$ E. Yablonovitch, Phys. Rev. Lett. 58, 2059 (1987).

${ }^{3}$ M. Maldovan and E. L. Thomas, Nature Mater. 3, 593 (2004)

${ }^{4}$ K. M. Ho, C. T. Chan, C. M. Soukoulis, R. Biswas, and M. Sigalas, Solid State Commun. 89, 413 (1994).

${ }^{5}$ S. H. Park, D. Qin, and Y. Xia, Adv. Mater. (Weinheim, Ger.) 10, 1028 (1998).

${ }^{6}$ M. Deubel, G. V. Freymann, M. Wegener, S. Pereira, K. Busch, and C. M. Soukoulis, Nature Mater. 3, 444 (2004).

${ }^{7}$ M. Campbell, D. N. Sharp, M. T. Harrison, R. G. Denning, and A. J. Turberfield, Nature (London) 404, 53 (2000).

${ }^{8}$ Y. V. Miklyaev, D. C. Meisel, A. Blanco, G. V. Freymann, K. Busch, W. Koch, C. Fnkrich, M. Deubel, and M. Wegener, Appl. Phys. Lett. 82, 1284 (2003).

${ }^{9}$ L. Wu, Y. Zhong, C. T. Chan, K. S. Wong, and G. P. Wang, Appl. Phys. Lett. 86, 241102 (2005).

${ }^{10}$ Y. Zhong, L. Wu, H. Su, K. S. Wong, and H. Wang, Opt. Express 14, 6837 (2006).

${ }^{11}$ Y. K. Pang, J. C. Lee, C. T. Ho, and W. Y. Tam, Opt. Express 14, 9113 (2006).

${ }^{12}$ Y. Lin, P. R. Herman, and K. Darmawikarta, Appl. Phys. Lett. 86, 071117 (2005).

${ }^{13}$ T. Y. M. Chan, O. Toader, and S. John, Phys. Rev. E 71, 046605 (2005).

${ }^{14}$ O. Toader, T. Y. M. Chan, and S. John, Appl. Phys. Lett. 89, 101117 (2006)

${ }^{15}$ Y. Lin, A. Harb, D. Rodriguez, K. Lozano, D. Xu, and K. P. Chen, Opt. Express 16, 9165 (2008).

${ }^{16}$ N. Tétreault, G. V. Freymann, M. Deubel, M. Hermatschweiler, F. P. Willard, S. John, M. Wegener, and G. A. Ozin, Adv. Mater. (Weinheim, Ger.) 18, 457 (2006).

${ }^{17}$ D. Xu, K. P. Chen, K. Ohlinger, and Y. Lin, Appl. Phys. Lett. 93, 031101 (2008) 